\title{
As culturas do confinamento: um olhar da crise, a partir da realidade dos povos indígenas
}

\section{- Confinement cultures: a look at the crisis, based on the reality of indigenous peoples}

\author{
Antonio Hilario Aguilera Urquiza' \\ Ana Keila Mosca Pinezi
}

\begin{abstract}
Resumo: O presente artigo objetivou desenvolver breve reflexão a respeito de como os povos indígenas brasileiros têm lidado com a pandemia pelo novo coronavírus e como têm sido afetados por ela. Ainda, pretendeu-se discutir o descaso do Estado brasileiro com os direitos humanos dessas populações mais vulneráveis. Em termos metodológicos, foram utilizadas pesquisas acerca do avanço do novo coronavírus entre etnias indígenas brasileiras, além de dados secundários de depoimentos em sites de ONGs e institutos especializados no assunto. A pesquisa nos mostra que a realidade da pandemia escancarou o quanto a sociedade brasileira é desigual em vários níveis e diferentes contextos, e como são tratadas deficientemente as questões de saúde de grupos vulneráveis, como os indígenas. Evidenciou, ainda, que não apenas cada comunidade tem sua maneira própria de conviver com a pandemia, mas também de que não há políticas de saúde adequadas e eficazes voltadas aos grupos vulneráveis que são os que mais sofrem perdas com esta crise sanitária e humanitária.
\end{abstract}

Palavras chave: Pandemia. Saúde indígena. Direitos humanos. Coronavírus.

1 Doutor em Antropologia de Iberoamérica pela Universidade de Salamanca, Espanha. Professor da Universidade Federal de Mato Grosso do Sul (UFMS), Brasil. hilarioaguilera@gmail.com

2 Doutora em Psicologia pela Universidade de São Paulo (USP). Professora da Universidade Federal do Triangulo Mineiro (UFTM), Brasil. keipinezi@gmail.com 


\begin{abstract}
This article aimed to develop a brief reflection on how different Brazilian indigenous groups deal with the pandemic caused by the new coronavirus, and how they have been affected by it. Furthermore, it intended to discuss the human rights' neglect by the Brazilian State against those very vulnerable populations. In terms of methodology, the approach taken was based on evaluation of research data about the advancement of the new coronavirus among Brazilian indigenous ethnicities, in addition to secondary testimonial data from NGOs' and subject matter specialized institutes' websites. This research shows evidence of how the pandemic clearly revealed the inequalities within Brazilian society, in various levels and different contexts, and how deficiently handled health issues are among vulnerable populations, like the indigenous groups. In addition, it indicates that not only each community has its own way of dealing with the pandemic, but also that there are no adequate and effective health policies aimed at vulnerable groups, which are the ones that suffer the most from this health and humanitarian crisis.
\end{abstract}

Keywords: Pandemic. Indigenous health. Human rights. Coronavirus.

\title{
Introdução
}

Escrever acerca do que está ocorrendo na atualidade - a crise humanitária causada pela Covid-19, declarada pandemia pela OMS (fevereiro de 2020) - é um grande desafio e ainda maior quando buscamos escrever em meio aos fatos e à avalanche de consequências provocados pelo caos na área da saúde, da economia e da política. Queremos, aqui, desenvolver uma breve reflexão, a partir da Antropologia e da forma como diferentes culturas enfrentam de formas diversas as adversidades. "La cultura no es la única variable humana existente con influencia en nuestras vidas, pero sí quizá una de las que nos proporciona mayor comprehensividad sobre las mismas" (ESPINA BARRIO, 2020, 12).

A impressão é que no Brasil vivemos essa crise de forma mais intensa que em outras regiões do planeta, tendo em vista a agudização também da crise política, em que no momento mais delicado na vida do país o Estado encontra-se sem um ocupante do cargo de ministro da saúde, o qual pelo menos poderia representar a direção de uma política pública em resposta aos desafios colocados pela crise sanitária.

Imaginemos esse contexto vivido por um grupo minoritário e discriminado pelo Estado brasileiro há mais de 500 anos, como é o caso dos povos indígenas. Notícias atuais dão conta de que um terço das etnias do país está com casos de infecção pelo novo coronavírus, em localidades de difícil acesso 
e precário atendimento da SESAl ${ }^{3}$.

A proposta deste texto, diante desse panorama, é refletir antropologicamente sobre a crise sanitária atual a partir da realidade dos povos indígenas, particularmente da do povo Guarani, no Estado de Mato Grosso do Sul, o qual possui a segunda maior população indígena do país. Ainda, pretendeuse discutir o descaso do Estado brasileiro com os direitos humanos dessas populações mais vulneráveis.

Em termos metodológicos, foram utilizadas pesquisas acerca do avanço do novo coronavírus entre etnias indígenas brasileiras, além de dados secundários de depoimentos em sites de ONGs e institutos especializados no assunto.

\section{O povo Guarani e o confinamento}

Os Guarani ${ }^{4}$, na atualidade, estão entre as cinco maiores etnias do Brasil, e fazem parte da família linguística tupi-guarani. Subdividem-se em três grupos: os Mbya, (mais encontrados no litoral do Espírito Santo ao Rio Grande do Sul), os Ñandeva e os Kaiowá.

Coube ao governador do Paraguai, Domingos Martinez Irala ${ }^{5}$, a partir de 1548, oficializar a "descoberta" dos Guarani dessa região, conhecidos então como da Província do Itatim. Grupos dessa província foram reduzidos pelos jesuítas a partir de 1632, com o argumento de protegê-los dos "encomenderos", exatamente na região onde atualmente se encontram os atuais Guarani Kaiowá (BRAND, 1997).

Esses Itatim do início do Período Colonial seriam os mesmos Caaguá (ou índios Monteses) do século XVIII, com novos nomes (reconceitualizados) dados pela colonização (bandeirantes). Com a expulsão dos jesuítas em 1768, esses indígenas ficaram sem referências e "este fato certamente aumentou as fugas para o mato, reforçando o contingente Guarani encontrado na re-

3 SESAI (Secretaria de Saúde Indígena) - Objetivo: a proteção, a promoção e a recuperação da saúde dos povos indígenas e exercer a gestão de saúde indígena, bem como orientar o desenvolvimento das ações de atenção integral à saúde indígena e de educação em saúde segundo as peculiaridades, o perfil epidemiológico e a condição sanitária de cada DSEI, em consonância com as políticas e programas do SUS (BRASIL, 2011).

4 No texto referimo-nos ao povo Guarani englobando sempre os Kaiowá e Ñandeva, sabendo que estes são mais conhecidos como "Guarani”, pois essa é a forma de auto identificação dada pelos integrantes do próprio grupo. Quando utilizado o termo "Kaiowá e Guarani” queremos incluir na argumentação ambos os povos (Guarani Kaiowá e Guarani Nandeva), ressaltando que apesar de falarem línguas muito próximas e de apresentarem aspectos sociais, culturais e econômicos similares, se constituem como povos diferentes e afirmam a sua diferença em diversos momentos oportunos como em disputas políticas internas nas Reservas e Terras Indígenas (AGUILERA URQUIZA \& PRADO, 2015, p. 50-51).

5 Domingos Martinez de Irala, foi Governador do Paraguai no século XVI. Em 1544 viaja de Assunção ao Guairá (nome pelo qual vai ser conhecida grande parte do atual Paraná/Brasil), para apresar índios para as encomiendas (forma de trabalho compulsório indígena), antes da catequização dos jesuítas 
gião da divisa Brasil/Paraguai" (BRAND, 1997, p. 53). Após esse período, esses grupos se embrenharam nas matas da região do então sul de Mato Grosso e foram impactados apenas com o início dos conflitos da Guerra entre Brasil e Paraguai, na segunda metade do século XIX.

Com o Governo de Getúlio Vargas e a política de "Marcha para o Oeste", foi incentivada a migração massiva para essa região, considerada "um vazio demográfico" e que precisava ser ocupada e colonizada. No início dos anos de 1940, foi criado o Território Federal de Ponta Porã e a Colônia Agrícola de Dourados, e foram tituladas a quase totalidade das terras, tidas como "devolutas", para os colonos.

Anos antes, o Governo Federal, através do SPI (Serviço de Proteção ao Índio) realizou a demarcação de 8 reservas, com algo em torno de 2 a $3 \mathrm{mil} \mathrm{h/a,} \mathrm{com} \mathrm{a}$ intenção de acomodar os Guarani dispersos, confinando-os nessas áreas, para meIhor controle e para a liberação do restante do território para a colonização. Muitos pesquisadores, como Brand (1997), chamam a esse movimento de um violento processo de confinamento: a expropriação dos territórios tradicionais dos povos indígenas e seu subsequente controle, através da oferta de saúde e educação.

Quanto, nos tempos atuais, refletimos sobre a condição de vivermos "confinados", devido à exigência do distanciamento social para conter o avanço do Covid-19, e pensamos que esses povos indígenas vivem essa experiência há cem anos? Vivem em fragmentos dos territórios tradicionais de seus ancestrais, sem espaço para caça, pesca e coleta de seu sustento. Vivem na dependência de políticas públicas quase sempre desrespeitosas de seus direitos humanos básicos.

Foi em meio a essa realidade de marginalização, preconceito veiculado pela mídia, superpopulação nas aldeias, invasão de igrejas pentecostais e neopentecostais, fragmentação de seus modelos de organização social e práticas culturais, altos índices de violência, alcoolismo e consumo de outras drogas, que no final do mês de maio de 2020 a contaminação pelo coronavírus chegou às aldeias Guarani de Mato Grosso do Sul ${ }^{6}$.

\section{Na Amazônia e no Mato Grosso do Sul - a pandemia entre os indígenas}

6 "A vulnerabilidade desses povos à covid-19 se agrava com o desmonte das políticas de saúde indígena e a desastrosa condução das ações pelo governo durante a pandemia. 'Os povos indígenas foram muito afetados pela descontinuidade no Ministério da Saúde’, critica o procurador Delfino de Almeida, que ajuizou uma ação civil para obrigar a Secretaria Especial de Saúde Indígena, do Ministério da Saúde, a fazer o mínimo frente à Covid-19: entregar equipamentos de proteção às equipes de saúde que atendem os guarani em Dourados” Repórter Brasil, junho/2020, Disponível em: https://reporterbrasil.org.br/2020/06/dos-frigorificos-as-plantacoes-de-cana-como-o-agronegocio-expos-indigenas-a-covid-19/?utm_campaign=shareaholic\&utm_medium=twitter\&utm_source=socialnetwork 
Uma vez que a contaminação chega às aldeias, tanto da região amazônica, sobretudo no Alto Rio Solimões (Município de Tabatinga/AM), como no Mato Grosso do Sul, em especial na região de Dourados, a crise humanitária se repete e se alastra, vitimizando ainda mais aqueles que já são discriminados e desrespeitados em seus direitos fundamentais, descritos na própria Constituição Federal de 1988, em seu artigo 231:

São reconhecidos aos índios sua organização social, costumes, línguas, crenças e tradições, e os direitos originários sobre as terras que tradicionalmente ocupam, competindo à União demarcá-las, proteger e fazer respeitar todos os seus bens.

Além do direito a seus territórios originários, são direitos conquistados o reconhecimento de sua organização social, costumes, língua, crenças e tradições. No entanto, sistematicamente, o Estado se nega a respeitar direitos básicos, sobretudo em relação ao atendimento diferenciado no âmbito da saúde indígena.

A crise atual tem mostrado a importância de termos no Brasil um sistema público de saúde, o qual, apesar de suas falhas e de ter sofrido ataques sistemáticos nos últimos governos, segue mostrando seu valor e o de seus milhares de funcionários: profissionais da área da saúde, técnicos e gestores. Nunca o papel do Estado foi tão importante e valorizado, inclusive pelos defensores do neoliberalismo e do Estado mínimo. Ao lado da importância do SUS (Sistema Único de Saúde), a pandemia expõe cruelmente e de forma escancarada as desigualdades sociais e nelas os grupos mais fragilizados, pois ela não afeta igualmente a população, como mostram estudos como o de Werneck e Carvalho (2020). Assim, os povos indígenas, dada sua situação marginal na sociedade brasileira, podem ser classificados como grupo de risco para Covid-19. De acordo com carta publicada na Revista Science, isso se explica por que

Indigenous and traditional peoples can be expected to be especially vulnerable to severe acute respiratory syndrome-coronavirus 2 (SARS-CoV-2), the virus that causes COVID-19. In addition to their history of susceptibility to epidemics, many of these isolated communities lack medical posts, doctors, and basic medications, to say nothing of the ventilators that would be needed to treat a COVID-19 outbreak. Bolsonaro's administration recently dismissed 8000 Cuban doctors who served small communities in the country's interior, which has been especially harmful to Indigenous and traditional communities in the Amazon region (FERRANTE \& FEARNSIDE, 2020, p. 251).

Segundo dados da Articulação de Povos Indígenas (APIB), uma organização que compila dados da SESAI (Secretaria Especial de Saúde Indígena), do Comitê Nacional de Vida e Memória Indígena e das Secretarias Municipais e Estaduais de Saúde e Ministério Público Federal sobre a infecção por Covid-19 
entre os indígenas, em 30 de junho de 2020 havia 8722 indígenas infectados, 377 mortos e 120 povos indígenas afetados ${ }^{7}$.

O indicador da fragilidade indígena, composto por análise dos dados de vulnerabilidade social, disponibilidade de leitos hospitalares, números de casos por município, número de óbitos, perfil etário da população indígena, vias de acesso e outros fatores relacionados com a estrutura de atendimento da saúde indígena e mobilidade territorial, mostra-se, segundo o ISA (Instituto Socioambiental), em patamar elevado no que tange ao Covid-19.

As últimas informações dão conta de que a pandemia avança sobre as aldeias indígenas de Mato Grosso do Sul, especialmente os Guarani, reforçando o estigma e o preconceito, por um lado, e por outro, aumentando a fome e a desolação fruto das mortes causadas pela Covid-19. Estudos apontam também que

\begin{abstract}
A população indígena em zona urbana reside majoritariamente em municípios com alto risco para COVID-19, totalizando 190.767 indígenas nessa situação. Corresponde a $67,5 \%$ da população indígena urbana do Centro-Oeste e 79,4\% da região Sul-Sudeste (FIOCRUZ, $4^{\circ}$ Relatório 2020, p. 3).,
\end{abstract}

Mesmo com a elaboração de pequenos livretos ${ }^{8}$ em língua guarani, explicando sobre o vírus, seu modus operandi e consequências para as aldeias, assim como evitar o contágio, muitas famílias não conseguem entender a periculosidade do mesmo, assim como há pessoas infectadas que se recusam a ser internadas em hospitais nas cidades e seguem nas aldeias infectando outros familiares.

Outra dificuldade é a concepção de "ficar em casa", prática que não faz parte da tradição indígena. Para muitos desses povos, a casa sempre muito pequena, é um lugar para dormir. As pessoas passam o dia ao ar livre, buscando alimentos. No caso dos povos indígenas, são culturas da coletividade, passam quase todo o dia em interações intensas. Como propor para essas famílias que devem ficar em casa? Situação semelhante constatou-se em muitas favelas, nas periferias das grandes cidades: famílias numerosas vivendo em espaços minúsculos, o que inviabiliza a prática do afastamento social.

Concretamente, nos casos dos indígenas do centro sul do Brasil, inúmeros casos de contaminação pela Covid-19 ocorreram em razão de empresas que seguiram funcionando, mesmo durante a quarentena, como o caso dos frigoríficos em Mato Grosso do Sul. Muitas aldeias tiveram seus primeiros

7 Disponível em: https://covid19.socioambiental.org/.

8 Trata-se de material paradidático, elaborado como subsídio de informação, nesse momento de crise sanitária, fruto da ação voluntária de professores/as (indígenas e não indígenas) da Ação Saberes Indígenas na Escola, um projeto financiado pelo MEC, até o final de 2018, e atualmente sem recursos do governo federal. Mesmo assim, muitos/as seguem mobilizados nas aldeias e cidades e seguem trabalhando dentro dessa proposta. 
surtos de contaminação, através de indígenas trabalhadores nesses frigoríficos, que retornaram com a doença para suas aldeias.

\section{Especificidades culturais, sociabilidade e a pandemia entre os povos indígenas.}

A vulnerabilidade dos povos indígenas é indiscutível. Os dados e as condições a que estão submetidos deixam isso patente: são mais elevados que na população brasileira os níveis de desnutrição, diarreia e anemia em crianças e mulheres indígenas. Estas últimas também apresentam altos índices de sobrepeso, obesidade, diabetes e anemia, segundo dados do Primeiro Inquérito $\mathrm{Na}$ cional de Saúde e Nutrição dos Povos Indígenas (COIMBRA et al., 2013).

Essas precárias condições de saúde estão vinculadas ao desrespeito aos territórios indígenas, a invasões que geram contaminações ambientais, a questões referentes à sustentabilidade alimentar e atenção à saúde, conforme aponta o $4^{\circ}$ Relatório (2020). ${ }^{9}$

O que poderíamos nos perguntar, como o fez Buchillet (2004), é justamente como essa população entende uma epidemia ou uma pandemia, no caso da Covid-19: como a iminência da doença ou a própria doença é vista por eles em termos geracionais, de gênero, de tratamentos tradicionais e nãotradicionais e como pensam, especificamente, a quarentena, algo que os obrigaria a desvincular-se, mesmo que temporariamente, da coletividade, o que é fundamental na cosmovisão e convívio deles. Outro complicador se impõe nesse caso: como saber o que pensam os indígenas sem que haja o diálogo por meio de entrevistas, grupos focais, etnografia ou quaisquer outros métodos e técnicas de pesquisa desse tipo, uma vez que o isolamento no caso da pandemia é condição essencial para conter o avanço da doença?

Lembramos aqui que a nosologia indígena não costuma ser a mesma das equipes de saúde, como bem aponta Buchillet (2004, p. 60) em seu estudo:

[...] Vale salientar que as percepções dos índios acerca da situação de saúde comunitária e das causas eventuais de sua melhora ou piora podem não coincidir com aquelas dos profissionais de saúde. Além das concepções divergentes em matéria de saúde, doença e cura, os profissionais de saúde podem se preocupar com uma doença não categorizada como doença pela comunidade, não reconhecida como sendo de competência da medicina ocidental ou, ainda, não

94 (o Relatório - 8 de abril - Risco de espalhamento da Covid-19 em populações indígenas: considerações preliminares sobre vulnerabilidade geográfica e sociodemográfica. Abril de 2020. Núcleo de Métodos Analíticos para Vigilância Epidemiológica do PROCC/Fiocruz e EMAp/FGV e GT sobre Vulnerabilidade Sociodemográfica e Epidemiológica dos Povos Indígenas no Brasil à Pandemia de Covid-19 e colaboradores. 
percebida como problema prioritário de saúde nessa comunidade específica.

Compreender, portanto, como os indígenas lidam com a doença, como a classificam, ao que ou a quem a atribuem é uma das etapas primeiras para implementar, em trabalho relacional e conjunto, formas de combatê-la. A utilização das pesquisas etnográficas sobre essa questão já realizadas pode oferecer subsídios para as políticas de saúde pública entre os indígenas em situações como a que vivenciamos com a pandemia.

Voltamos à questão do isolamento social, fundamental para evitar o espalhamento da infecção por Covid-19. Sabe-se que a coletividade entre os povos indígenas é característica cultural marcante. Pensando o processo saúde/doença como um construto sociocultural é necessário que a atenção biomédica leve em consideração as práticas locais e as peculiaridades culturais. Assim, no caso dos povos indígenas, a noção de isolamento social precisa ser pensada a partir do olhar deles e quais as soluções que eles sugerem diante dessa situação. Dessa maneira, o foco não é mais o profissional de saúde, mas os atores sociais envolvidos e, no caso específico dos indígenas, a coletividade. Evocamos aqui o conceito de práticas de "autoatenção" definidas como "as representações e práticas que a população utiliza no nível do sujeito e do grupo social para diagnosticar, explicar, atender, controlar, aliviar, aguentar, curar, solucionar ou prevenir os processos que afetam sua saúde em termos reais ou imaginários, sem a intervenção direta, central e intencional de curadores profissionais" (MENÉNDEZ, 2009, p. 48). A autonomia é o fundamento desse conceito, mas que pressupõe a troca de conhecimentos, em que há influências mútuas no processo constante de construção e reconstrução de modelos de saúde. Daí, então, surgem práticas terapêuticas combinadas que não estão acabadas ou fechadas em um modelo biomédico, mas que são fruto de trocas fundamentadas em respeito mútuo e reconhecimento das peculiaridades culturais. Para isso, portanto, é indispensável a participação de indígenas na gestão dos serviços de saúde, algo já bastante discutido e reivindicado pelos próprios indígenas.

Sobre essa questão, a da autoatenção, Cardoso (2015, p. 101) destaca que:

Garnelo (2001) salienta, por exemplo, que entre os Baniwa o discurso "autorizado" sobre saúde é apropriado pela cultura local, que seleciona, dentre o conjunto de informações transmitidas, os elementos que apresentam tanto "relações de congruência com os saberes preexistentes", tanto quanto aqueles que podem "subsidiar e legitimar as práticas e demandas políticas e sociais" atuais da população nativa.

Cardoso (2015) mostra, em sua pesquisa, a trajetória da política de 
saúde indígena no Brasil e que houve um movimento com o objetivo de ultrapassar a dicotomia, vinda do modelo biomédico, entre conhecimento científico positivista e o reconhecimento do complexo conhecimento nativo das peculiaridades culturais e as práticas locais. Esse movimento foi feito por meio da criação de subsistemas de saúde nomeados Distritos Sanitários Especiais Indígenas (DSEls), incorporados ao SUS, que foram criados em 1999. Em tese, os conselhos distritais e a comunidade local teriam o "controle social" na gestão de políticas públicas em saúde, que substituiria o modelo clássico sanitarista. Entre vários pontos do projeto, os DSEls deveriam promover a formação de Agentes Indígenas de Saúde (AISs) para que atuassem em suas próprias comunidades. Os DSEls seriam unidades gestores autónomas. Contudo, houve, com a oficialização da SESAI em 2010, vinculado ao Ministério da Saúde, uma "departamentalização burocrática" e uma gestão centralizada na "organização administrativa dos DSEls" (CARDOSO, 2015) em detrimento da autonomia indígena no que se refere à prestação de serviços em atenção em saúde à população indígena. Ainda, Cardoso mostra os problemas na formação dos agentes de saúde indígenas cujo projeto pedagógico, pautado no modelo biomédico, pretendia marcar as diferenças de concepção de saúde e doença entre indígenas e não-indígenas, inferiorizando os saberes nativos.

\begin{abstract}
Observamos os instrutores procurando explicar aos Agentes Indígenas que "o coração é que faz funcionar o corpo", que "cada corpo é um corpo (separado e distinto dos demais)", que o uso da "camisinha não irá sufocar a criança", entre outras "informações" que procuram marcar a diferença entre "crenças", a serem "respeitadas" e o "conhecimento científico", aquilo que comprovadamente se "sabe" e deve ser transmitido para a "produção da saúde" (CARDOSO, 2015, p. 100).
\end{abstract}

Segundo Cardoso (2015, p. 89), "o modelo assistencial implantado nos distritos segue a lógica da produção de serviços, centrado na concepção médico-curativa e na tecnificação da assistência", sem conseguir atender às necessidades dessa população. Além do "controle social", segundo aponta Cardoso (2015), os indígenas entendem a noção de autonomia também no plano político na gestão de políticas de saúde, da qual foram alijados, apesar do documento que concebe a "Política Nacional de Atenção à Saúde Indígena" prever justamente o contrário:

Com o objetivo de garantir o acesso à atenção de média e alta complexidade, deverão ser definidos procedimentos de referência, contrarreferência e incentivo a unidades de saúde pela oferta de serviços diferenciados com influência sobre o processo de recuperação e cura dos pacientes indígenas (como os relativos a restrições/ prescrições alimentares, acompanhamento por parentes e/ou intérprete, visita de terapeutas tradicionais, instalação de redes, entre outros) quando considerados necessários pelos próprios usuários e 
negociados com o prestador de serviços (BRASIL, 2000, p. 15).

Não é escopo deste artigo explorar a trajetória de como se constituiu institucionalmente a política de saúde indígena no Brasil, mas apontar que a "autoatenção" indígena, que envolve autonomia e controle político e social nas políticas públicas de atenção à saúde, não se concluiu, mesmo com as DSEls, o que poderia, nesta pandemia, mudar o panorama da situação de vulnerabilidade dos povos indígenas frente a essa crise humanitária.

Ainda são vistos como crenças, em oposição à ciência ocidental, elementos dos sistemas (complexos) indígenas no que se refere à saúde e doença, ao corpo, às terapias e ao autocuidado, por exemplo. Como bem apontou Mendonça (2005, p. 09), há desigualdades de poder no encontro dessas visões de mundo:

De um lado a sociedade industrializada; de outro, sociedades indí-
genas. De um lado um sistema médico "moderno" no qual, segundo
Clavreul (1978), "a doença definida, de acordo com o ideal democrá-
tico, é semelhante em todos os homens e está excluída radicalmen-
te da influência de forças inobjetíveis, isto é, separadas do domínio
da religião e da magia. Dessa forma, a prática da medicina ocidental
é estabelecida à custa da negação da singularidade tanto individual
como social do indivíduo, em benefício da afirmação da universa-
lidade do homem". De outro lado, a medicina tradicional na qual a
doença, e principalmente o estar doente, é inscrita no conjunto das
representações do homem, de suas atividades em sociedade e de
seu meio natural. Para estas, a doença não existe fora de seu contex-
to sociocultural e, portanto, de um contexto singular.

Assim, a supremacia da sociedade envolvente é reforçada em relação aos povos indígenas, uma vez que o modelo biomédico, ainda hegemônico, ao desqualificar o sistema de conhecimentos indígena obstrui o entendimento do universo cultural do outro e o possível diálogo/articulação no processo de formação dos agentes de saúde indígenas e de novas conformações terapêuticas. Em lugar desse modelo biomédico fechado usado no processo terapêutico propõe-se o que a antropologia médica sob a perspectiva interpretativa-crítica advoga:

Quando emprega a abordagem crítica-interpretativa, a antropologia médica não é mais o estudo de sistemas médicos alternativos, crenças e práticas... O enfoque é mudado para examinar a maneira em que todo o conhecimento relacionado ao corpo, saúde e doença é construído culturalmente, negociado e renegociado num processo dinâmico através do tempo e do espaço (LOCK; SCHEPER-HUGHES, 1990, p. 49; apud LANGDON, 1994, p. 116).

Entendendo a doença como "[...] um processo "experiencial" - suas manifestações dependem de fatores culturais, sociais e psicológicos, operando conjuntamente com processos psicobiológicos" (LANGDON, 1994, p. 115), é 
possível afirmar que o enfrentamento de uma pandemia, como a que vivemos, provocada pela Covid-19, necessariamente precisa ser pensado com base no significado que esse evento tem dentro dos incontáveis universos culturais que não são estáticos, mas fluidos, e tampouco homogêneos e que, abertos como são, reconstroem-se por meio da interação social concreta.

Em relação aos povos indígenas, não se pretende restringir a situação de vulnerabilidade deles aos problemas do sistema de atenção de saúde indígena. Há outras dimensões correlacionadas, já aqui apontadas, que contribuem para essa situação como o avanço do agronegócio em terras indígenas e o trabalho de indígenas em lavouras e em empresas como as frigoríficas. Em 24 de junho de 2020, matéria do Repórter Brasil ${ }^{10}$ mostra que houve o registro da primeira morte por Covid-19 de um indígena da Reserva Indígena de Dourados. Segundo a reportagem, o coronavírus entrou na aldeia por meio de uma funcionária da JBS, frigorífico onde trabalhava. Houve afastamento de funcionários indígenas de frigoríficos como a JBS, mas isso demorou a acontecer, conforme noticiado na reportagem. Erileide Domingues, uma das indígenas, que trabalha nas 20 barreiras das aldeias Guarani e Kaiowá, diz que não consegue barrar a saída dos que vão trabalhar nas plantações ou em setores terceirizados do agronegócio: Alguns saem para trabalhar... Se estiver contaminado, volta e acaba contagiando seis ou doze pessoas que estão dentro de casa.

Erileide explica "que as lideranças defendem, desde o início da pandemia, o afastamento temporário dos trabalhadores indígenas, tanto das lavouras quanto dos frigoríficos" (REPÓRTER BRASIL, 2020). Ainda segundo essa reportagem,

\begin{abstract}
Para Tonico Benites, antropólogo indígena e membro da Aty Guasu, grande assembleia dos povos kaiowá e guarani, houve descaso da JBS com as comunidades indígenas. Ele quer que empresas e governo federal sejam responsabilizados pelo contágio e está acionando o Ministério Público Federal neste sentido. A Comissão Guarani Yvyrupa também registrou denúncia junto ao MPT e ao MPF, cobrando medidas de proteção para os trabalhadores indígenas e as comunidades guarani.
\end{abstract}

Como se vê, há inúmeros aspectos que estão envoltos na condição de vulnerabilidade dos indígenas que envolvem questões políticas, sociais e de reconhecimento identitário. A pandemia pelo novo coronavírus deixou essa condição escancarada e despiu o modelo tradicional biomédico expondo-o como ineficaz e ultrapassado para lidar com saúde/doença em contextos multiétnicos.

10 Dos frigoríficos às plantações de cana: como o agronegócio expôs indígenas à Covid-19. Disponível em: https://reporterbrasil.org.br/2020/06/dos-frigorificos-as-plantacoes-de-cana-como-o-agronegocio-expos-indigenas-a-covid-19/?utm_campaign=shareaholic\&utm_medium=twitter\&utm_source=socialnetwork. Acesso em: 26 jun. 2020. 


\section{O "bem viver" - o que aprender dos indígenas}

Os indígenas sempre defenderam o direito a viver em um meio ambiente saudável, o que está garantido, inclusive no ordenamento jurídico internacional, em especial na Convenção 169 da OIT (Organização Internacional do Trabalho de 1989). O primeiro elemento importante para o "bem viver", na concepção desses povos tradicionais, é exatamente a relação harmoniosa com a natureza, entendida aqui não apenas em seu aspecto material, mas, sobretudo, espiritual.

Assim como estamos percebendo em nossa sociedade ocorre de forma ainda mais aguda com os povos indígenas: a crise provocada por essa pandemia traz consigo um profundo "mal-estar", desestabilizando não apenas as relações entre humanos, mas a relação consigo mesmo e com o cosmos. Imaginemos o desconforto para essas comunidades tradicionais que não podem realizar seus rituais, inclusive xamanísticos, para afastar os perigos e proteger a coletividade.

O direito em ver respeitada sua língua e cultura é outro elemento fundamental para a qualidade de vida desses povos indígenas, garantido no artigo 231 da Constituição Federal de 1988, além de ser sinal de valorização da cultura e identidade indígena. No entanto, no contexto da pandemia em que estamos vivendo, o mais importante talvez seja o direito em ter uma assistência à saúde que leve em consideração códigos e práticas culturais dos indígenas.

A crise provocada mundialmente pela Covid-19 demonstra que cada cultura tem suas concepções próprias do que seja saúde, doença e prevenção. Para os povos indígenas, saúde não é apenas ausência de enfermidades, ou "comorbidades" (palavra que entrou na moda agora), mas é o "bem viver", em uma situação de equilíbrio e harmonia, que eles chamam em sua língua "teko porã", que traduzida literalmente seria "o viver bonito". Assim, mais um elemento importante quando falamos em direitos humanos é a sensibilidade em respeitar as particularidades culturais, em especial no momento de cuidado com o corpo e a saúde das pessoas.

\section{Considerações finais}

Falar em direitos humanos, durante a pandemia causada pelo novo coronavírus e a partir da Antropologia, leva-nos a ampliar o olhar para outros horizontes, aqueles que nos posicionam para fora da nossa cultura, na possibilidade de outras compreensões do mundo e do próprio contexto que nos assola. Neste sentido, nosso ponto de referência foi a situação dos povos 
indígenas, os quais estão entre as principais vítimas nesse momento de crise sanitária. Enquanto estão recolhidos em suas aldeias, seus territórios tradicionais são invadidos, como é o caso do povo Yanomami, por madeireiros e garimpeiros, os quais além da violência ambiental, ainda transmitem o vírus letal para os indígenas. Diante da omissão do Governo Federal, eles são os mais vulneráveis, pois enquanto a taxa de letalidade da população brasileira para a Covid-19 é de 5,6\%, a indígena chega a 9,6\%, conforme dados da APIB (Associação dos Povos Indígenas no Brasil), conforme opinião no Jornal Folha de São Paulo .

Não poder cuidar dos seus mortos ou serem obrigados a cumprir protocolos de afastamento social que não compreendem, pois, toda a vida deles apenas tem significado no social, no coletivo, são situações limites, em especial para os povos indígenas, mas também para todos nós, o que nos oferece a oportunidade para repensarmos nosso modelo de vida consumista/individualista. O respeito à dignidade humana é um compromisso de todos.

\section{Referências}

AGUILERA URQUIZA, Antonio Hilario; PRADO, José Henrique. O impacto do processo de territorialização dos Kaiowá e Guarani no sul de Mato Grosso do Sul. Tellus, Campo Grande-MS, ano 15, n. 29, p. 49-71, jul. /dez. 2015.

BRAND, Antonio J. O impacto da perda da terra sobre na tradição Kaiowá/Guarani: os difíceis caminhos da palavra. Tese (Doutorado em História) - Pontifícia Universidade Católica do Rio Grande do Sul, Porto Alegre. 1997.

BRASIL. Fundação Nacional de Saúde. Política Nacional de Atenção à Saúde dos Povos Indígenas. Brasília: Ministério da Saúde; Fundação Nacional de Saúde, 2000.

BRASIL. Ministério da Saúde/Secretaria Especial de Saúde Indígena. Ministério da Saúde/Secretaria Especial de Saúde Indígena, 2011.

BUCHILLET, D. Cultura e saúde pública: reflexões sobre o Distrito Sanitário Especial Indígena do Rio Negro. In: LANGDON, E. J.; GARNELO, L. (org.). Saúde dos povos indígenas: reflexões sobre antropologia participativa. RJ: ABA, 2004. p. 53- 67.

CARDOSO, Marina. Políticas de saúde indígena no Brasil: do modelo assistencial à representação política. In: LANGDON, E. J.; CARDOSO, M. D. Saúde indígena: políticas comparadas na América Latina. Florianópolis: Ed. da UFSC, 
2015. p. 83-106.

COIMBRA, C. E. A., et al. The First National Survey of Indigenous People's Health and Nutrition in Brazil: Rationale, methodology, and overview of results. BMC Public Health, 13 (1), 52. 2013.

ESPINA BARRIO, Ángel Baldomero. Pandemias y culturas: diversas formas socio-culturales de afrontar las desgracias. Revista Interdisciplinaria de Estudios Latinoamericanos - RIEL. Comitán de Domínguez, Chiapas (México), v. 4, n. 2, p. 11-18, jun. 2020.

FERRANTE, L.; FEARNSIDE, P. M. Protect Indigenous peoples from COVID-19. Science, Washington - DC, v. 368, Issue 6488, p. 251, 17 apr. 2020. Disponível em: https://science.sciencemag.org/content/368/6488/251.1. Acesso em: 26 jun. 2020.

FIOCRUZ (Fundação Oswaldo Cruz). $4^{\circ}$ relatório sobre risco de espalhamento da COVID-19 em populações indígenas. 2020. Disponível em: https://portal. fiocruz.br/documento/4oretorio-sobre-risco-de-espalhamento-da-covid-19-em-populaçoes-indíginas. Acesso em: 26 de jun. 2020

ISA - Instituto Socioambiental. COVID19 entre os indígenas. Disponível em: https://covid19.socioambiental.org/. Acesso em: 26 jun. 2020.

LANGDON, E. J. Representações de doença e itinerário terapêutico entre os Siona da Amazônia Colombiana. In: SANTOS, R. V.; COIMBRA, C. (org.). Saúde e povos indígenas. Rio de Janeiro: Fiocruz, 1994. p. 115-142.

MENDONÇA, S. B. M. O agente indígena de saúde no Parque Indígena do Xingu: reflexões. In: BARUZZI, R.; JUNQUEIRA, C. (org.). Parque Indígena do Xingu: saúde, cultura e história. São Paulo: Unifesp: Terra Virgem, 2005. p. 227-244.

MENÉNDEZ, E. L. Sujeitos, saberes e estruturas: uma introdução ao enfoque relacional no estudo da saúde coletiva. São Paulo: HUCITEC, 2009.

PIMENTEL, Spensy Kamitta. Elementos para uma teoria política kaiowá e guarani. Dissertação de Mestrado, Antropologia Social da USP/SP, 2012, 375 p.

REPÓRTER BRASIL. Dos frigoríficos às plantações de cana: como o agrone- 
gócio expôs indígenas à Covid-19. Por Nataly Foscaches e Tatiane Klein | 24/06/20. Disponível em: https://reporterbrasil.org.br/2020/06/dos-frigorificos-as-plantacoes-de-cana-como-o-agronegocio-expos-indigenas-a-covid-19/?utm_campaign=shareaholic\&utm_medium =twitter\&utm_source=socialnetwork. Acesso em: 26 jun. 2020.

WERNECK, G. L. \& CARVALHO, M. S. A pandemia de COVID-19 no Brasil: uma crise sanitária anunciada. Cadernos de Saúde Pública, Rio de Janeiro, 36(5), p.1-4, 2020. Disponível em: https://www.scielosp.org/pdf/csp/2020.v36n5/ e00068820/pt. Acesso em: 26 jun.2020. 
